\title{
Home-based devices in dermatology: a systematic review of safety and efficacy
}

\author{
Marc Cohen $^{1} \cdot$ Evan Austin ${ }^{1} \cdot$ Natasha Masub $^{1} \cdot$ Alana Kurtti $^{2,3} \cdot$ Christopher George $^{1} \cdot$ Jared Jagdeo $^{1,2} \mathbb{C}$
}

Received: 1 February 2021 / Revised: 22 March 2021 / Accepted: 8 April 2021 / Published online: 3 May 2021

(c) The Author(s) 2021

\begin{abstract}
There is increasing demand for home-based devices for the treatment of dermatologic conditions and cosmesis. Commercially available devices include intense pulsed light, laser diodes, radiofrequency, light-emitting diodes, and ultraviolet B phototherapy. The objective of this report is to evaluate the current evidence regarding the efficacy and safety of home-based devices for the treatment of skin conditions. A systematic search of PubMed, Embase, and Cinahl was conducted on November 9, 2020 using PRISMA guidelines. Original research articles that investigated the efficacy and safety of home-based devices for dermatologic use were included. Bibliographies were screened for additional relevant articles. Strength of evidence was graded using the Oxford Centre for Evidence-Based Medicine guidelines. Clinical recommendations were then made based on the quality of the existing literature. After review, 37 clinical trials were included -19 were randomized controlled trials, 16 were case series, and 2 were non-randomized controlled trials. Ultimately, from our analysis, we recommend the home-based use of intense pulsed light for hair removal, laser diodes for androgenic alopecia, low power radiofrequency for rhytides and wrinkles, and light-emitting diodes for acne vulgaris. Trials investigating ultraviolet B phototherapy for psoriasis revealed mixed evidence for home treatments compared to clinic treatments. All devices had favorable safety profiles with few significant adverse events. Limitations to our review include a limited number of randomized controlled trials as well as a lack of data on the long-term efficacy and safety of each device.
\end{abstract}

Keywords Home devices $\cdot$ Hair removal $\cdot$ Alopecia $\cdot$ Light therapy $\cdot$ Psoriasis $\cdot$ Wrinkles

\section{Introduction}

In recent years, dermatology has witnessed a major transition to home-based care for some cosmetic and medical problems [23]. Undesirable body hair, androgenic alopecia, acne, skin aging, and psoriasis are among the conditions with treatments that can be done at home. While these conditions were traditionally treated by dermatologists in a clinical setting, they often required frequent visits and expensive therapies [2,3]. Given their low cost and convenience,

Jared Jagdeo

jrjagdeo@gmail.com

1 Department of Dermatology, State University of New York, SUNY Downstate Medical Center, 450 Clarkson Ave, Brooklyn, NY 11203, USA

2 Dermatology Service, VA New York Harbor Healthcare System Brooklyn Campus, Brooklyn, NY, USA

3 Rutgers Robert Wood Johnson Medical School, Piscataway, NJ, USA home-based therapies are increasing in popularity [3]. For example, the hair removal industry, a $\$ 9$ billion market, now consists largely of home treatments, including home devices, waxing, and depilatories [3]. The home medical equipment market accounted for $\$ 30.54$ billion in 2019 , and is estimated to reach $\$ 56.45$ billion by 2027 , with therapeutic equipment being the highest contributor [24]. Due to consumer demand, numerous Food and Drug Administration (FDA) approved products are currently on the market for home use in dermatology, many of which are light-based devices [23].

The recent advent of teledermatology may accelerate this transition to home-based care [8]. A recent review identified 229 dermatology-related mobile apps available to consumers [6]. This increase in teledermatology illustrates an emerging direction of dermatologic care that provides more patients with access to dermatologists from home [8]. We anticipate home-based devices becoming increasingly relevant as a consequence of this transition. This systematic review aims to summarize the existing literature on home-based devices 
in dermatology and provide evidence-based clinical recommendations on their efficacy and safety.

\section{Methods}

A systematic search was performed on November 9, 2020 using PubMed, Embase, and Cinahl, according to the Preferred Reporting Items for Systematic Reviews and Meta-analysis (PRISMA) guidelines. The search terms home use or home device were combined with the terms integumentary system, dermatology, low-level light therapy, photobiomodulation, phototherapy, skin, hair, alopecia, nails, wrinkle, aging, rejuvenation, visible light, psoriasis, or ultraviolet (Fig. 1). Search results were then screened for inclusion by two authors independently. Bibliographies of included articles were screened for additional relevant articles. Disputes between reviewers about whether a specific article fulfilled inclusion criteria had to be resolved with unanimous agreement after discussion for that article to be selected for this review. Clinical trials pertaining to the efficacy and safety of home-based devices for dermatologic indications were selected for inclusion. Studies were conducted in one of two ways: patients selftreated at home or were treated by medical professionals in a clinical setting using device parameters intended for home use. Studies that did not investigate a dermatologic indication and studies in which treatment was not intended for home use were excluded. Conference abstracts, literature reviews, poster presentations, non-English articles,
Fig. 1 PRISMA search strategy—search conducted according to preferred reporting items for systematic reviews and meta-analysis (PRISMA) procedure
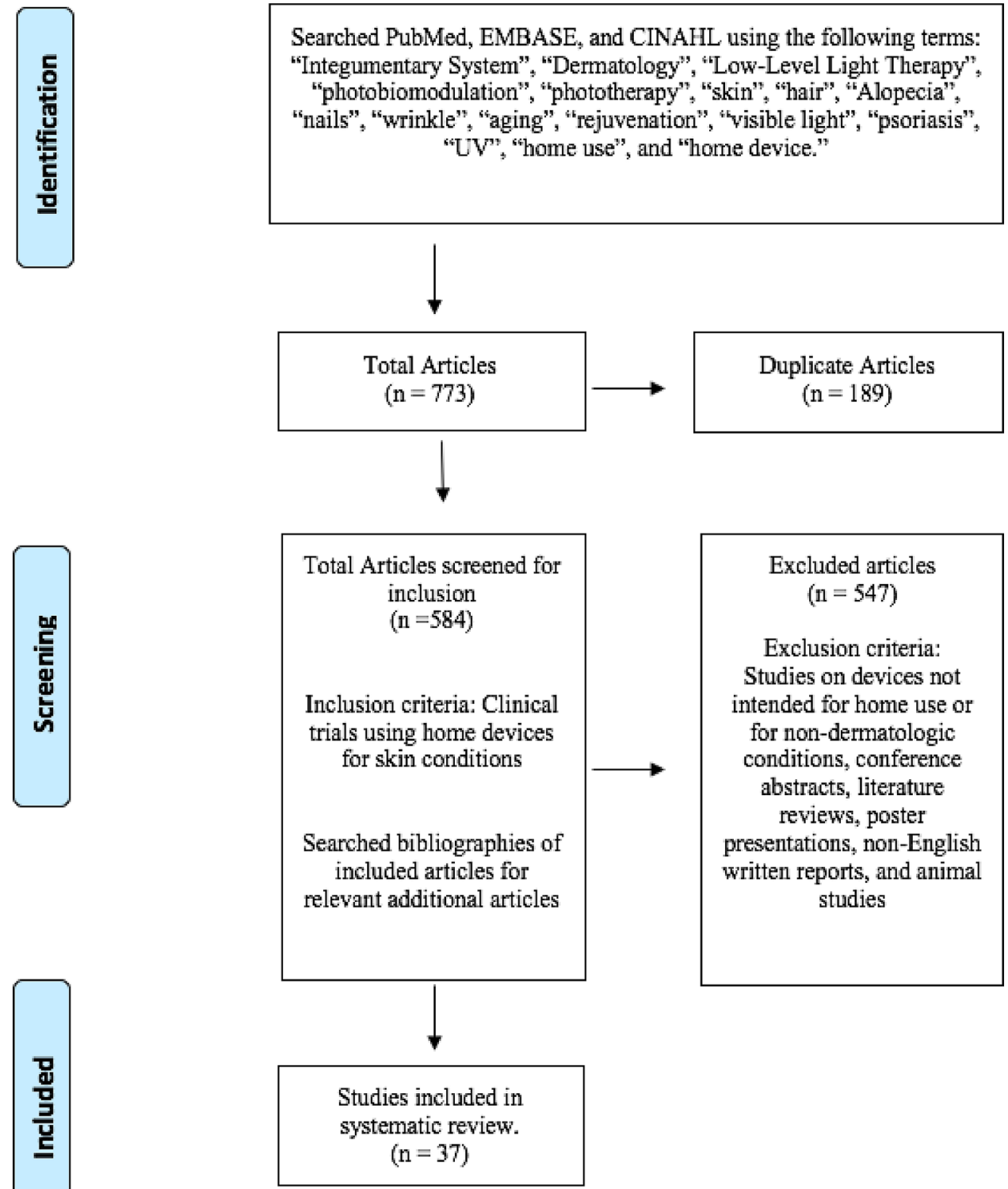

Excluded articles $(n=547)$

Exclusion criteria: Studies on devices not intended for home use or for non-dermatologic conditions, conference abstracts, literature reviews, poster presentations, non-English written reports, and animal studies 
laboratory investigations, and animal studies were also excluded.

\section{Results}

We identified 670 articles regarding home use devices. After screening titles, abstracts, and full texts, 37 studies involving 1,871 patients were identified that met inclusion criteria, including 19 randomized controlled trials (RCTs), 16 case series, and 2 non-randomized controlled trials (non-RCTs). Herein, we will generally limit our discussion to RCTs identified for each home-based modality. Table I details the study design, results, and adverse effects for all studies included in this review. Level of evidence (Table 1) and grades of recommendation (Table 2) were graded using the Oxford Centre for Evidence-Based Medicine guidelines. Table 3

Table 1 Levels of evidence by study

\begin{tabular}{llc}
\hline Level of evidence & $\begin{array}{l}\text { Total number of } \\
\text { studies }\end{array}$ & Included studies \\
\hline $1 \mathrm{~b}$ & 16 & $2,3,12,13,21,25$, \\
& & $26,27,28,30,31$, \\
& & $36,38,39,40,41$ \\
$2 \mathrm{~b}$ & 19 & $4,5,7^{\mathrm{a}}, 11,15,17$, \\
& & $18,19,22,29,32$, \\
& & $34,35,37,42,43$, \\
& 3 & $7^{\mathrm{a}}, 9,16$ \\
\hline
\end{tabular}

Level of study is determined by Oxford Centre for Evidence-based Medicine guidelines

${ }^{\text {a Study }} 7$ is included twice because it involved 2 phases of different study design displays device names, manufacturers, and indications for studies referenced in the results section.

\section{Intense pulsed light for hair removal}

Intense pulsed light (IPL) for hair removal involves photothermolysis of hair follicles [20]. Specifically, non-coherent light of broad wavelengths $(500-1200 \mathrm{~nm})$ is absorbed by melanin of the hair bulb and follicle [14]. Four RCTs investigated the efficacy of IPL for hair removal $[2,3,30$, 41]. One study utilized three biweekly treatments with IPL and resulted in a $53.6 \%$ decrease in hair count at 6 months compared to baseline. Mild erythema and follicular edema were reported side effects in $25 \%$ of patients [3]. Another RCT investigating the same IPL device reported a $64 \%$ decrease in hair count at 3 months after the same treatment regimen with minimal adverse events [30]. In another IPL study involving axillary hair treatments once weekly for four weeks, there was an $87 \%$ decrease in hair count at 6 months versus control. All 10 subjects experienced a mild burning/ itching sensation after administration [41]. Lastly, an RCT investigating IPL demonstrated home IPL was more efficacious and tolerable than conventional hot waxing. Mild erythema was reported [2].

Grade of Recommendation: A for the included home IPL devices for hair removal based on 5 level $1 \mathrm{~b}$ studies, 2 level $2 \mathrm{~b}$ studies, and 1 level 4 study (see Table 2).

\section{Laser diodes for androgenic alopecia}

Red and near-infrared lasers can prolong the anagen growth phase of the hair follicle [38]. We identified 5 RCTs evaluating the use of home-based laser devices for androgenic alopecia $[13,21,25,28,38]$. One study investigating low-level
Table 2 Grades of recommendations

\begin{tabular}{lll}
\hline Treatment & $\begin{array}{l}\text { Grade of recommen- } \\
\text { dation }\end{array}$ & Number of studies by evidence level \\
\hline IPL for hair removal & A & 5 level 1b studies [2, 3, 30, 40, 41] \\
& & 2 level 2b studies [11, 17] \\
Laser for androgenic alopecia & A & $\begin{array}{l}\text { 5 level 4 study [9] } \\
1 \text { level 1b studies [13, 21, 25, 28, 38] }\end{array}$ \\
RF for wrinkles & B & 1 level 1b study [31]
\end{tabular}

Grades of recommendations: A is based on consistent level 1 studies. B is based on consistent level 2 or 3 studies

$I P L$ Intense pulsed light, $R F$ Radiofrequency, $L E D$ Light emitting diode, $U V B$ Ultraviolet $\mathrm{B}$ 
Table 3 Device information for included studies in results section

\begin{tabular}{lllll}
\hline Study number & Technology & Use & Device name & Manufacturer/location \\
\hline 3.30 & IPL & Hair removal & Silk'n home & Home Skinovations, Kfar Saba, Israel \\
41 & IPL & Hair removal & iPulse Personal & CyDen Ltd, Swansea, UK \\
2 & IPL & Hair removal & E-One device & E-Swin, Paris, France \\
13 & Laser & Androgenic alopecia & HANDI-DOME & Capillus, Miami, FL \\
21 & Laser & Androgenic alopecia & HairMax LaserComb & Lexington international, Boca Raton, FL \\
38 & Laser & Androgenic alopecia & RAMACAP & Division of dermatology, Ramathibodi hospital, \\
& & & & Bangkok, Thailand \\
25 & Laser & Androgenic alopecia & Oaze & Won technology, Daejeon, South Korea \\
28 & Laser & Androgenic alopecia & TOPHAT655 & Apira science Inc., Boca Raton, FL \\
31 & RF & Wrinkles & Derma Wand & ICTV brands, Wayne, PA \\
34 & RF & Wrinkles & NEWA & EndyMed medical, Caesera, Israel \\
27 & LED & Acne vulgaris & O'cimple Light Therapy System MP 200 & Ceragem medisys Inc., Cheonan, Korea \\
36 & LED & Acne Vulgaris & no!no! & Radiancy incorporated, Orangeburg, NY \\
18 & LED & Acne vulgaris & Tanda Zap & Pharos life corporation, Ontario, Canada \\
26 & UVB & Psoriasis & Waldmann UV-100 & Waldmann, Villingen-Schwenningen, Germany \\
12 & UVB & Psoriasis & Dermasun Helios & Dermasun medical BV, Amsterdam, Netherlands \\
42 & UVB & Psoriasis & Clarify Home Light Therapy System & Clarify medical, San Diego, CA \\
\hline
\end{tabular}

This table denotes device name, study number, technology, indication, and manufacturing information

$I P L$ Intense Pulsed Light, $R F$ Radiofrequency, LED Light Emitting Diode, UVB Ultraviolet $\mathrm{B}$

laser devices demonstrated a 51\% increase in hair count after laser treatments for 30 min every other day for 17 weeks compared to sham-treatments. No adverse effects were reported [13]. Another study similarly demonstrated a significant increase in terminal hair count post laser treatment relative to the control. No adverse effects were reported [21]. A study investigating laser therapy on 40 patients showed a significant increase in hair density in the laser-treated group compared to the control group. One patient reported mild hair shedding and 2 patients reported scalp pruritus [38]. Additionally, a separate RCT evaluating laser treatments once daily for 24 weeks demonstrated significantly increased mean hair density compared to the sham-treated group. Of 20 patients, 9 reported minor headache and 5 reported skin pain, pruritus, or erythema [25]. Lastly, an RCT exploring laser therapy demonstrated a 39\% increase in hair count in the treatment group compared to the sham-treated group. No side effects were reported [28].

Grade of recommendation: A for the included home LED and laser devices for androgenic alopecia based on 5 level $1 \mathrm{~b}$ studies and 1 level $2 \mathrm{~b}$ study (see Table 2).

\section{Radiofrequency for rhytides and wrinkles}

Radiofrequency (RF) devices stimulate fibroblast collagen production [4]. We identified 1 RCT of home-based RF for rhytides and wrinkles [31]. RF led to a maximum reduction in eyebrow to hairline distance of $1.521 \mathrm{~cm}$ after a single treatment. No adverse effects were reported [31]. In a related
non-RCT, a home-based RF device demonstrated significant improvement in tactile elasticity and skin firmness. 45 patients received treatment 5 times weekly for 4 weeks followed by maintenance therapy twice weekly for 7 weeks. All 45 participants reported the treatment to be painless with only mild transient erythema as a side effect [34].

Grade of Recommendation: B for the included home RF devices for rhytides and wrinkles based on 1 level $1 \mathrm{~b}$ study and 6 level $2 \mathrm{~b}$ studies (see Table 2).

\section{Light emitting diode for acne vulgaris}

Blue light (BL) light-emitting diodes (LEDs) activate endogenous porphyrins produced by $C$. acnes which induce free radical production resulting in bacterial cell death [18]. Red light (RL), in contrast, is believed to have an anti-inflammatory effect, attenuating cytokine release by macrophages [19]. Three RCTs investigated home-based LED devices for the treatment of acne $[18,27,36]$. One study revealed a significant decrease in inflammatory and non-inflammatory acne lesions by $77 \%$ and $54 \%$, respectively, in 35 LEDtreated patients for 2.5 min twice daily for 4 weeks. Two patients reported mild skin dryness and 1 patient experienced erythema and desquamation [27]. Another RCT demonstrated a significant decrease in time required for acne lesion resolution compared to the sham irradiated placebo. No adverse effects were reported [36]. Lastly, a study of a different LED device showed significantly decreased lesion 
size and degree of erythema in the treated lesions versus placebo. No adverse effects were reported [18].

Grade of recommendation: B for the included home LED devices for acne vulgaris based on 2 level $1 \mathrm{~b}$ studies, 3 level $2 \mathrm{~b}$ studies, and 1 level 4 study (see Table 2).

\section{Ultraviolet Phototherapy for Psoriasis}

UVB phototherapy has demonstrated clinical efficacy for the treatment of psoriasis. The mechanism is hypothesized to involve induction of apoptosis in hyperproliferative cells, suppression of the immune response, and modification of the cytokine milieu [46]. Four RCTs evaluating home UVB phototherapy for psoriasis were included $[12,26,32]$. One study involving 3-4 treatments weekly for 3 months demonstrated a 74\% decrease in median Psoriasis Activity and Severity Index score (PASI) in the home treatment group compared to a $70 \%$ decrease in clinic-treated patients. $87 \%$ of participants experienced some degree of erythema while $6 \%$ reported mild skin blistering [26]. In a separate RCT consisting of 62 patients treated for 7 min daily for 6 months, there was a significant reduction in mean PASI score at 6 months from baseline in the treatment group compared to the control group. No adverse effects were reported [12]. Another trial studying home UVB exhibited a 57\% reduction in Psoriasis Severity Index (PSI) compared to no change in controls after 3 treatments weekly for 10 weeks. There were no adverse effects [42]. In contrast, in an RCT involving 40 patients, phototherapy (LISUP device) sessions given three times weekly for 8 weeks at home were compared to the same regimen provided in a clinical setting. 8 of 20 patients treated at home experienced complete clearance of psoriasis lesions compared to 18 of 20 clinic-treated patients who experienced complete clearing. Erythema was reported in both study arms [32]. The non-RCTs included in our review demonstrated efficacy for the home devices evaluated [7, $22,42,47]$.

Grade of recommendation: B for the included home phototherapy (UVB) devices for psoriasis based on 2 level $1 \mathrm{~b}$ studies, 4 level 2b studies, and 1 level 4 study (see Table 2).

\section{Discussion}

This analysis provides evidence for the safety and efficacy of several home-based devices for the treatment of dermatologic conditions. Home-based IPL devices demonstrated efficacy for hair removal. Reductions in hair count were consistent and exceeded $50 \%$ in all RCTs [2, 3, 30, 41]. The non-RCTs reviewed provided comparable findings. (Table I). While mild, transient erythema was the most commonly reported concern, none of the reviewed studies reported serious adverse effects $[2,3,9,11,17,30,40,41]$. Therefore, the home-based IPL devices identified should be regarded as safe therapeutic options for patients desiring at-home hair removal. It should be noted that for darker-skinned patients, low-fluence IPL is preferred as previous research has demonstrated optimal safety at this dose [9]. Ultimately, we recommend the use of the reviewed IPL home devices for patients seeking an affordable, safe, and efficacious home hair removal therapy. The reviewed studies do not, however, provide enough evidence to recommend specific treatment parameters. Additionally, it is worth noting that the American Academy of Dermatology (AAD) has not issued specific guidelines addressing home IPL for hair removal. For completeness sake, 3 studies examining home laser devices for hair removal were included in Table I [39, 43, 44]. While laser hair removal is commercially very popular, its use for this purpose with home devices, although promising, is less well studied.

The use of home-based laser devices for the treatment of androgenic alopecia has grown over the last 15 years [38]. While vasodilator minoxidil and 5-alpha reductase inhibitor finasteride are current mainstay medical therapies, they are not effective in all patients [38]. Home-based laser devices for androgenic alopecia demonstrated increased hair growth in subjects with minimal adverse effects. Therefore, we recommend home use of this therapeutic modality with the identified devices. However, this recommendation is limited by the significant variation in treatment parameters, as adjunct LED therapy was used in some of the reviewed studies [25, 28, 38]. Therefore, a specific recommendation regarding treatment parameters and regimens cannot be made. Adverse effects were present in 3 studies but were not reported to be severe or of major concern [25, 37, 38]. For example, one patient treated with the RAMACAP device experienced minor hair shedding and two reported scalp itching (of 40 total subjects) [38]. No specific guidelines have been issued by the AAD addressing home laser devices for androgenic alopecia.

Home-based RF devices also demonstrated efficacy and a favorable safety profile for the treatment of rhytides and wrinkles. In addition to the RF studies cited in the results section, non-RCT clinical studies also exhibited statistically significant improvement in wrinkle volume and skin laxity $[4,5]$. We selected a B recommendation for the RF devices included in this review. Recommended parameters include power outputs between 10 and $12 \mathrm{~W}$ for treatments every other day for 1-2 months. No specific guidelines have been issued by the AAD addressing home RF for wrinkle treatment.

Home-based LED devices identified in our systematic review demonstrated safety and efficacy in treating acne and received an A recommendation. In addition to the clinical studies included in our review, there is substantial basic science evidence (in vitro and in vivo studies) supporting the use of 
LEDs for acne treatment, and thus home devices are promising options for therapy [33]. We recommend home BL or RL LEDs with power densities of $6-40 \mathrm{~mW} / \mathrm{cm}^{2}$ and $8-80 \mathrm{~mW} /$ $\mathrm{cm}^{2}$, respectively, and treatment regimens of 2-3 times weekly for 3-6 weeks. These suggested parameters reflect the treatment regimens identified by this review. The current AAD clinical guidelines, however, indicate that there is limited evidence to recommend the use and benefit of physical modalities, such as LED, for the routine treatment of acne [1].

The use of UV phototherapy in psoriasis has dramatically changed the landscape of psoriasis treatment options [26]. A significant drawback of UV phototherapy, however, is that it often requires numerous clinic visits which can be burdensome for patients [26]. Thus, home-based UVB phototherapy may present a convenient therapeutic option for psoriasis patients. Our systematic review identified conflicting evidence for the efficacy of home-based UVB compared to traditional clinic-based administration. In one RCT, there was statistically significant PASI score reduction with home use compared to clinic treatments while another study revealed the opposite outcome [26, 32]. Other nonrandomized studies investigating home-based UVB phototherapy in psoriasis also demonstrated clinical efficacy. While the current literature leads to a $\mathrm{B}$ recommendation for home-based UVB phototherapy devices for psoriasis, the favorable safety profile and proof of efficacy may permit dermatologists to offer this therapeutic option for patients with medication refractory disease who prefer home treatment. However, there is insufficient evidence to make specific treatment regimen suggestions at this time. Our overall $B$ recommendation for home-based UVB coincides with that of the AAD guidelines [10].

The major limitation of our review is the lack of doubleblinded RCTs evaluating home-based devices for dermatologic treatment. Thus, many of the included studies (19 of 37) were not RCTs. While these studies do provide meaningful insights into the efficacy of these devices, they may not provide the same strength of evidence as RCTs. Additionally, most of the studies included in our review were conducted over a period of months, and thus the long-term efficacy of the devices remains uncertain. To further our understanding of the subject, double-blinded, placebo-controlled RCTs should be conducted. In addition, future studies should include long-term follow-ups to investigate the longterm effects of home-based dermatologic treatments as well as the need for maintenance treatments.

\section{Conclusion}

Home-based devices represent the future of dermatologic treatment for a multitude of conditions given their efficacy, safety, cost-effectiveness, and convenience. We determined that home-based devices are efficacious and safe for a number of dermatologic conditions, including IPL for hair removal, laser diodes for androgenic alopecia, RF for rhytides and wrinkles, and LED-BL/RL for acne. Conflicting evidence exists regarding phototherapy for home treatment of psoriasis. All treatment modalities demonstrated favorable safety profiles. Dermatologists should consider these home-based devices to address patients' dermatologic needs.

Supplementary Information The online version contains supplementary material available at https://doi.org/10.1007/s00403-021-02231-0.

Acknowledgements We would like to express our gratitude to the SUNY Downstate Department of Dermatology and SUNY Downstate Medical Center for their support in the completion of this study.

\section{Declarations}

Conflict of interest The authors declare that they have no conflict of interest.

Availability of data and material All data included in this review is available in published journals.

Open Access This article is licensed under a Creative Commons Attribution 4.0 International License, which permits use, sharing, adaptation, distribution and reproduction in any medium or format, as long as you give appropriate credit to the original author(s) and the source, provide a link to the Creative Commons licence, and indicate if changes were made. The images or other third party material in this article are included in the article's Creative Commons licence, unless indicated otherwise in a credit line to the material. If material is not included in the article's Creative Commons licence and your intended use is not permitted by statutory regulation or exceeds the permitted use, you will need to obtain permission directly from the copyright holder. To view a copy of this licence, visit http://creativecommons.org/licenses/by/4.0/.

\section{References}

1. Acne clinical guideline (2020) https://www.aad.org/member/clini cal-quality/guidelines/acne.

2. Adhoute $\mathrm{H}$, Hamidou Z, Humbert P, Lyonnet C, Peuchot MA, Reygagne P, Reynier C, Rivoire S, Simoneau G, Toubel G (2010) Randomized study of tolerance and efficacy of a home-use intense pulsed light (IPL) source compared to the hot-wax method. J Cosmet Dermatol 9:287-290. https://doi.org/10.1111/j.1473-2165. 2010.00523.x

3. Alster TS, Tanzi EL (2009) Effect of a novel low-energy pulsedlight device for home-use hair removal. Dermatol Surg 35:483489. https://doi.org/10.1111/j.1524-4725.2009.01089.x

4. Beilin G (2011) Home-use TriPollar RF device for facial skin tightening: clinical study results. J Cosmet Laser Ther 13:69-76. https://doi.org/10.3109/14764172.2011.552607

5. Boisnic S, Branchet MC, Birnstiel O, Beilin G (2010) Clinical and histopathological study of the TriPollar home-use device for body treatments. Eur J Dermatol 20:367-372. https://doi.org/10. 1684/ejd.2010.0938

6. Brewer AC, Endly DC, Henley J, Amir M, Sampson BP, Moreau JF, Dellavalle RP (2013) Mobile applications in dermatology. 
JAMA Dermatol 149:1300-1304. https://doi.org/10.1001/jamad ermatol.2013.5517

7. Cameron H, Yule S, Moseley H, Dawe RS, Ferguson J (2002) Taking treatment to the patient: development of a home TL-01 ultraviolet B phototherapy service. Br J Dermatol 147:957-965. https://doi.org/10.1046/j.1365-2133.2002.04860.x

8. Campagna M, Naka F, Lu J (2017) Teledermatology: an updated overview of clinical applications and reimbursement policies. Int J Womens Dermatol 3:176-179. https://doi.org/10.1016/j.ijwd. 2017.04.002

9. Elm CM, Wallander ID, Walgrave SE, Zelickson BD (2010) Clinical study to determine the safety and efficacy of a low-energy, pulsed light device for home use hair removal. Lasers Surg Med 42:287-291. https://doi.org/10.1002/1sm.20917

10. Elmets CA, Lim HW, Stoff B, Connor C, Cordoro KM, Lebwohl M, Armstrong AW, Davis DMR, Elewski BE, Gelfand JM, Gordon KB, Gottlieb AB, Kaplan DH, Kavanaugh A, Kiselica M, Kivelevitch D, Korman NJ, Kroshinsky D, Leonardi CL, Lichten J, Mehta NN, Paller AS, Parra SL, Pathy AL, Farley Prater EA, Rupani RN, Siegel M, Strober BE, Wong EB, Wu JJ, Hariharan V, Menter A (2019) Joint american academy of dermatology-national psoriasis foundation guidelines of care for the management and treatment of psoriasis with phototherapy. J Am Acad Dermatol 81:775-804. https://doi.org/10.1016/j.jaad.2019.04.042

11. Emerson R, Town G (2009) Hair removal with a novel, low fluence, home-use intense pulsed light device. J Cosmet Laser Ther 11:98-105. https://doi.org/10.1080/14764170902792199

12. Franken SM, Witte B, Pavel S, Rustemeyer T (2015) Psoriasis and daily low-emission phototherapy: effects on disease and vitamin D level. Photodermatol Photoimmunol Photomed 31:83-89. https:// doi.org/10.1111/phpp.12151

13. Friedman S, Schnoor P (2017) Novel approach to treating androgenetic alopecia in females with photobiomodulation (low-level laser therapy). Dermatol Surg 43:856-867. https://doi.org/10. 1097/dss.0000000000001114

14. Gold M (2007) Lasers and light sources for the removal of unwanted hair. Clin Dermatol 25:443-453. https://doi.org/10. 1016/j.clindermatol.2007.05.017

15. Gold MH, Biron J, Levi L, Sensing W (2017) Safety, efficacy, and usage compliance of home-use device utilizing RF and light energies for treating periorbital wrinkles. J Cosmet Dermatol 16:95-102. https://doi.org/10.1111/jocd.12299

16. Gold MH, Biron JA, Sensing W (2014) Clinical and usability study to determine the safety and efficacy of the silk'n blue device for the treatment of mild to moderate inflammatory acne vulgaris. J Cosmet Laser Ther 16:108-113. https://doi.org/10.3109/14764 172.2013.854638

17. Gold MH, Biron JA, Thompson B (2015) Clinical evaluation of a novel intense pulsed light source for facial skin hair removal for home use. J Clin Aesthet Dermatol 8:30-35

18. Gold MH, Sensing W, Biron JA (2011) Clinical efficacy of homeuse blue-light therapy for mild-to moderate acne. J Cosmet Laser Ther 13:308-314. https://doi.org/10.3109/14764172.2011.630081

19. Goldberg DJ, Russell BA (2006) Combination blue (415 nm) and red $(633 \mathrm{~nm})$ LED phototherapy in the treatment of mild to severe acne vulgaris. J Cosmet Laser Ther 8:71-75. https://doi.org/10. 1080/14764170600735912

20. Husain Z, Alster TS (2016) The role of lasers and intense pulsed light technology in dermatology. Clin Cosmet Investig Dermatol 9:29-40. https://doi.org/10.2147/ccid.S69106

21. Jimenez JJWT, Bergfeld W (2014) Efficacy and safety of a lowlevel laser device in the treatment of male and female pattern hair loss: a multicenter, randomized, sham device-controlled, doubleblind study. Am J Clin Dermatol 15:115-127. https://doi.org/10. 1007/s40257-013-0060-6
22. Jordan WP Jr, Clarke AM, Hale RK (1981) Long-term modified Goeckerman regimen for psoriasis using an ultraviolet B light source in the home. J Am Acad Dermatol 4:584-591. https://doi. org/10.1016/s0190-9622(81)70061-6

23. Juhász ML, Levin MK, Marmur ES (2017) A review of available laser and intense light source home devices: a dermatologist's perspective. J Cosmet Dermatol 16:438-443. https://doi.org/10. 1111/jocd.12371

24. Wadekar T, Sumant O (2020) Home Medical Equipment Market Expected to Reach $\$ 56.45$ Billion by 2027 . Available from: https://www.alliedmarketresearch.com/press-release/homecaremedical-equipment-market.html

25. Kim H, Choi JW, Kim JY, Shin JW, Lee S-j, Huh C-H (2013) Low-level light therapy for androgenetic alopecia: a 24 week, randomized, double-blind, sham device-controlled multicenter trial. Dermatol Surg 39:1177-1183. https://doi.org/10.1111/dsu.12200

26. Koek MB, Buskens E, van Weelden H, Steegmans PH, Bruijnzeel-Koomen CA, Sigurdsson V (2009) Home versus outpatient ultraviolet B phototherapy for mild to severe psoriasis: pragmatic multicentre randomised controlled non-inferiority trial (PLUTO study). BMJ 338:b1542. https://doi.org/10.1136/bmj.b1542

27. Kwon HH, Lee JB, Yoon JY, Park SY, Ryu HH, Park BM, Kim YJ, Suh DH (2013) The clinical and histological effect of home-use, combination blue-red LED phototherapy for mild-to-moderate acne vulgaris in Korean patients: a double-blind, randomized controlled trial. Br J Dermatol 168:1088-1094. https://doi.org/ 10.1111/bjd.12186

28. Lanzafame RJ, Blanche RR, Bodian AB, Chiacchierini RP, Fernandez-Obregon A, Kazmirek ER (2013) The growth of human scalp hair mediated by visible red light laser and LED sources in males. Lasers Surg Med 45:487-495. https://doi.org/10.1002/1sm. 22173

29. Leyden J, Stephens TJ, Herndon JH Jr (2012) Multicenter clinical trial of a home-use nonablative fractional laser device for wrinkle reduction. J Am Acad Dermatol 67:975-984. https://doi.org/10. 1016/j.jaad.2012.01.041

30. Mulholland RS (2009) Silk'nTM-a novel device using home pulsed lightTM for hair removal at home. J Cosmet Laser Ther 11:106-109. https://doi.org/10.1080/14764170902902806

31. Nobile V, Michelotti A, Cestone E (2016) A home-based eyebrows lifting effect using a novel device that emits electrostatic pulses containing RF energy, resulting in high frequency, low level transdermal microcurrent pulsations: double blind, randomized clinical study of efficacy and safety. J Cosmet Laser Ther 18:234-238. https://doi.org/10.3109/14764172.2016.1156704

32. Paul BS, Stern RS, Parrish JA, Arndt KA (1983) Low-intensity selective UV phototherapy. A clinical trial in outpatient therapy for psoriasis. Arch Dermatol 119:122-124

33. Rathi S (2011) Acne vulgaris treatment: the current scenario. Indian J Dermatol 56:7-13. https://doi.org/10.4103/0019-5154. 77543

34. Sadick NS, Harth Y (2016) A 12 week clinical and instrumental study evaluating the efficacy of a multisource radiofrequency home-use device for wrinkle reduction and improvement in skin tone, skin elasticity, and dermal collagen content. J Cosmet Laser Ther 18:422-427. https://doi.org/10.1080/14764172.2016.12024 19

35. Sadick NS, Harth Y, Levy H, Shemer A (2014) Home based wrinkle reduction using a novel handheld multisource phase-controlled radiofrequency device. J Drugs Dermatol 13:1342-1347

36. Sadick NS, Laver Z, Laver L (2010) Treatment of mild-to-moderate acne vulgaris using a combined light and heat energy device: home-use clinical study. J Cosmet Laser Ther 12:276-283. https:// doi.org/10.3109/14764172.2010.538409

37. Santino J, Markou M (2004) Hair regrowth and increased hair tensile strengthusing the hairmax lasercomb forlow-level laser 
therapy. Int J Cosmet Surg Aesthet Dermatol. https://doi.org/10. 1089/153082003769591209

38. Suchonwanit P, Chalermroj N, Khunkhet S (2019) Low-level laser therapy for the treatment of androgenetic alopecia in Thai men and women: a 24 week, randomized, double-blind, sham devicecontrolled trial. Lasers Med Sci 34:1107-1114. https://doi.org/10. 1007/s10103-018-02699-9

39. Thaysen-Petersen D, Barbet-Pfeilsticker M, Beerwerth F, Nash JF, Philipsen PA, Staubach P, Haedersdal M (2015) Quantitative assessment of growing hair counts, thickness and colour during and after treatments with a low-fluence, home-device laser: a randomized controlled trial. Br J Dermatol 172:151-159. https://doi. org/10.1111/bjd.13254

40. Thaysen-Petersen D, Erlendsson A, Nash J, Beerwerth F, Philipsen P, Wulf H, Haedersdal M, Erlendsson AM, Nash JF, Philipsen PA, Wulf HC (2015) Ultraviolet radiation after exposure to a low-fluence IPL home-use device: a randomized clinical trial. Lasers Med Sci 30:2171-2177. https://doi.org/10.1007/ s10103-015-1796-4

41. Trelles MA, Ash C, Town G (2014) Clinical and microscopic evaluation of long-term ( 6 months) epilation effects of the ipulse personal home-use intense pulsed light (IPL) device. J Eur Acad Dermatol Venereol 28:160-168. https://doi.org/10.1111/jdv.12069

42. Unrue EL, Cline A, Collins A, Nguyen VH, Pelle MT, Blake P, Feldman SR (2019) A novel ultraviolet B home phototherapy system: efficacy, tolerability, adherence, and satisfaction. Dermatol Online J 25(2)
43. Wheeland RG (2007) Simulated consumer use of a battery-powered, hand-held, portable diode laser $(810 \mathrm{~nm})$ for hair removal: a safety, efficacy and ease-of-use study. Lasers Surg Med 39:476493. https://doi.org/10.1002/lsm.20518

44. Wheeland RG (2012) Permanent hair reduction with a home-use diode laser: safety and effectiveness 1 year after eight treatments. Lasers Surg Med 44:550-557. https://doi.org/10.1002/lsm.22051

45. Wheeland RG, Koreck A (2012) Safety and effectiveness of a new blue light device for the self-treatment of mild-to-moderate acne. J Clin Aesthet Dermatol 5:25-31

46. Wong THL, Liao W (2013) Phototherapy in psoriasis: a review of mechanisms of action. J Cutan Med Surg 17:6-12. https://doi. org/10.2310/7750.2012.11124

47. Yentzer BA, Yelverton CB, Pearce DJ, Camacho FT, Makhzoumi Z, Clark A, Boles A, Fleischer AB Jr, Balkrishnan R, Feldman SR (2008) Adherence to acitretin and home narrowband ultraviolet B phototherapy in patients with psoriasis. J Am Acad Dermatol 59:577-581. https://doi.org/10.1016/j.jaad.2008.06.007

Publisher's Note Springer Nature remains neutral with regard to jurisdictional claims in published maps and institutional affiliations. 